\title{
Rectal ultrasound with fine needle aspiration: an underutilized modality for delineating and diagnosing perirectal, presacral, and pelvic lesions
}

다(1)이우

\author{
Authors \\ Landon K. Brown ${ }^{1}$, Norman R. Clark ${ }^{2}$, Jason Conway², Girish Mishra²
}

Institutions

1 Department of Internal Medicine, Wake Forest School of Medicine, Winston-Salem, North Carolina, United States

2 Department of Gastroenterology, Wake Forest School of Medicine, Winston-Salem, North Carolina, United States

submitted 6.4.2018

accepted after revision 25.7.2018

Bibliography

DOI https://doi.org/10.1055/a-0743-5356 |

Endoscopy International Open 2019; 07: E171-E177

(c) Georg Thieme Verlag KG Stuttgart · New York ISSN 2364-3722

Corresponding author

Dr. Girish Mishra, Wake Forest Baptist Medical Center, Medical Center Boulevard, Winston-Salem, NC 27157 gimishra@wakehealth.edu

\section{ABSTRACT}

Background and study aims The merits of rectal ultrasound for rectal cancer staging are well documented. Conventional approaches to accessing perirectal and presacral lesions entail computed tomography guidance via a transgluteal approach or frank surgical exploration. We report on the safety and efficacy of performing rectal ultrasound with fine-needle aspiration (RUS-FNA) for evaluating perirectal, presacral, and pelvic abnormalities.

Patients and methods Patients who underwent RUS-FNA of perirectal, presacral, or pelvic lesions between August 2005 and September 2016 were identified using an institutional database. Subjects were all individuals treated at Wake Forest Baptist Medical Center in Winston-Salem, North Carolina, United States. Patient demographics and imaging characteristics were noted. Procedural details included lesion size, location, echo appearance, and technical information. Patients were given antibiotics prior to FNA attempt and for 3 days after. Diagnostic yield, clinical utility, and complications were noted.

Results Twenty-seven patients met criteria during the specified study time period. The cohort consisted of 12 males (44.4\%) and 15 females (55.5\%). RUS-FNA was diagnostic in 24 patients $(88.8 \%)$ and obviated the need for surgery in 14 patients $(51.9 \%)$. There were four complications (14.8\%): two perirectal and two presacral abscesses.

Conclusion While the diagnostic yield of RUS-FNA is high and the potential to affect clinical decision-making is substantial, risk of complication is not negligible. RUS-FNA should only be performed if the result will substantially alter clinical management, and the decision to perform RUSFNA should be made with close consultation between the endosonographer, surgeon, and/or medical or radiation oncologist.

\section{Introduction}

Rectal cancer is one of the most commonly diagnosed malignancies with an estimated 40,000 new cases a year in the United States with reported local recurrence rates ranging from $3 \%$ to $9.2 \%$ after treatment [ $1-5]$. These recurrences, as well as other primary/malignant pathological lesions, can manifest as perirectal, presacral, and pelvic lesions. Rectal ultrasound (RUS), computed tomography (CT), and magnetic resonance imaging (MRI) have all been utilized to evaluate and stage these primary and recurrent rectal malignancies [6-10]. However, effective and safe tissue diagnosis in the perirectal, presacral, and pelvic lesions is crucial for effective management as these lesions can encompass a broad differential.

The ability to perform RUS fine-needle aspiration (FNA) allows for pathological confirmation that can have considerable clinical impact on managing patients. This modality has been used in diagnosing perirectal, pelvic, and urologic lesions [1115]. However, there is a paucity of data about the diagnostic yield and inherent risks of RUS-FNA compared to the conventional approaches of CT-guided transgluteal or surgical assessment of perirectal, presacral, and pelvic lesions. The purpose of this study was to evaluate the efficacy and safety of performing RUS-FNA for presacral, perirectal, and pelvic abnormalities. 


\section{Patients and methods}

This study was approved by the Institutional Review Board of Wake Forest Baptist Medical Center. Our retrospective case series used an institutional database to investigate patients who underwent RUS-FNA of perirectal, presacral, or pelvic lesions between August 2005 and September 2016. Twenty-seven patients met criteria during the specified study time period. Data including age, gender, prior imaging modalities utilized, pathology results, and outcomes were collected. All procedures were performed in an outpatient setting using moderate sedation by administering both midazolam and fentanyl or deep sedation with IV propofol by a licensed CRNA. All endoscopic procedures were performed using an Olympus UM130 or UM160 radial and linear echoendoscope with dopplers (Olympus America, Inc, Center Valley, Pennsylvania, United States). Furthermore, RUS-FNA was performed with a 22- or 25-gauge needle by experienced endosonographers at our tertiary referral center. All subjects received prophylactic ciprofloxacin $400 \mathrm{mg}$ prior to FNA and 3 days following the procedure.

\section{Results}

Our patient cohort consisted of 12 males (44.4\%) and $15 \mathrm{fe}-$ males (55.5\%) with an average patient age of 51 (range 1980). Information for each case is summarized in $>$ Table 1. Twelve patients (44.4\%) had known prior rectal or colon adenocarcinoma. One patient (3.7\%) had known endometriosis. All but one patient had prior imaging. A perirectal mass was detected at hysterectomy in the patient with no prior imaging. Imaging modalities included CT (22, 81.5\%), MRI (4, 14.8\%), and positron emission tomography $(12,44.4 \%)$. On imaging, a presacral mass was present in 12 patients (44.4\%), a perirectal node was present in two patients (7.4\%), a perirectal abnormality was present in 11 patients (40.7\%), and a pelvic mass was present in one patient (3.7\%). The average size of lesion present on imaging was $3.58 \mathrm{~cm}$ (range 0.9 to $16.0 \mathrm{~cm}$ ). Excluding the $16-\mathrm{cm}$ lesion that was too large to be measured on RUS, the average size of lesion recorded on RUS was $3.12 \mathrm{~cm}$ (range 0.9 to 7.6$)$. Eighteen lesions (66.7\%) were hypoechoic and nine lesions (33.3\%) were heterogeneous on RUS.

FNA pathology distribution was as follows: adenocarcinoma $(6,22.2 \%)$, squamous cell carcinoma $(2,7.4 \%)$, benign lymphoid hyperplasia (2,7.4\%), benign epithelial cells $(3,11.1 \%)$, benign atypical or nonspecific cells $(2,7.4 \%)$, benign reactive or inflammatory cells $(2,7.4 \%)$, myelolipoma $(1,3.7 \%)$, benign, cystic lesion (dermoid cyst, inclusion cyst, or teratoma) $(4,14.8 \%)$, non-diagnostic $(2,7.4 \%)$, sarcoma $(1,3.7 \%)$, seminal vesicle $(1,3.7 \%)$, urothelial bladder cancer (1,3.7\%). All adenocarcinomas were recurrent malignancies. RUS-FNA provided an effective diagnosis in 24 patients, giving a diagnostic yield of $88.8 \%$, and diagnosed recurrent adenocarcinoma in six patients.

RUS-FNA was non-diagnostic in three cases (11\%). In one case, a specimen was initially labeled benign-appearing cells, however, subsequent surgical pathology reported a cystic hamartoma. In a second case, aspecimen was initially labeled be- nign atypical glandular cells, however, subsequent surgical pathology determined the specimen to be endometriosis. In the third case, a specimen was incorrectly read by pathology as a gastrointestinal stromal tumor (GIST) but subsequent surgical pathology revealed the lesion to be endometriosis. Five individuals $(18.5 \%)$ were lost to follow-up.

We encountered four complications in two presacral and two perirectal mass FNAs ( $>$ Table 2 ). Our complication rate was approximately $25 \%$ with biopsies of presacral masses and $8 \%$ of the perirectal biopsies. No complications were observed with the pelvic mass RUS-FNA. The overall total complication rate was approximately $14.8 \%$ in the form of abscess formation requiring either drainage or surgical intervention. Average needle passes performed in the four cases with complications was 2.5 passes. The location of abscess formation coincided with the original biopsy site. The echo characteristics of the four lesions were as follows: two heterogeneous and two hypoechoic. All four individuals had benign cytopathology on FNA. One out of the four individuals had an extended hospital course requiring two different incision and drainage (I\&D) procedures and prolonged course of antibiotics because of an infected sacral teratoma. That individual subsequently improved after intervention but was lost to follow-up. The other individual with a presacral abscess had subsequent abscess excision with improvement of symptoms. The two individuals with perirectal abscesses both presented with fever and rectal pain. Both individuals underwent $I \& D$ with no reported complications. The two individuals' symptoms improved after treatment.

\section{Discussion}

RUS-FNA provides unparalleled ability to sample lesions surrounding the perirectal space including presacral and pelvic lesions. Previous studies have highlighted RUS-FNA's role in diagnosing local pelvic urologic malignancies/masses, in confirming nodal metastases in early rectal cancer, in accurately diagnosing perirectal lesions (CRC and other lesions), and in preventing aggressive surgical interventions for benign conditions [1216]. Our study is one of the larger descriptive cohort studies that highlights the clinical utility of RUS-FNA for assessing and accessing perirectal, presacral, and pelvic lesions. However, few have reported on diagnostic and safety data on RUS-FNA. Our study shows that RUS-FNA alters management in patients with perirectal, presacral, and pelvic lesions. Notably, our study had a diagnostic accuracy of $88.8 \%$ which coincided with previous reports of FNA procedures in perirectal, intraluminal, and pelvic lesions [15-17]. Surgery was avoided in $55.5 \%$ of our subjects and clinically impacted approximately $60 \%$ of subjects, indicating the importance of RUS-FNA's ability to obtain a tissue diagnosis and inform a decision about institution of medical and/or surgical therapy. These findings suggest that RUS-FNA is an accurate and useful clinical tool in management of patients with presacral, perirectal, and pelvic lesions.

Although RUS-FNA is relatively safe, we found a significantly higher complication rate. Approximately $15 \%$ of our patients developed an abscess. This higher complication rate is in stark contrast to the relatively uncommon reported complications 
- Table 1 Patient and clinical characteristics.

\begin{tabular}{|c|c|c|c|c|c|c|c|c|}
\hline & $\begin{array}{l}\text { Age/ } \\
\text { Sex }\end{array}$ & $\begin{array}{l}\text { Radiographic } \\
\text { findings }\end{array}$ & $\begin{array}{l}\text { Overall } \\
\text { U/S ap- } \\
\text { pearance }\end{array}$ & $\begin{array}{l}\text { Fine- } \\
\text { needle } \\
\text { gauge }\end{array}$ & $\begin{array}{l}\text { Pathology ob- } \\
\text { tained from FNA }\end{array}$ & $\begin{array}{l}\text { Compli- } \\
\text { cations }\end{array}$ & Outcome & $\begin{array}{l}\text { Surgery } \\
\text { avoided } \\
\text { (Yes/No) }\end{array}$ \\
\hline 1 & $43 / F$ & $\begin{array}{l}\mathrm{CT} \text { : presacral mass, } \\
\text { right hydronephrosis } \\
\mathrm{PET} \text { : pelvic enhance- } \\
\text { ment }\end{array}$ & $\begin{array}{l}\text { Hypoe- } \\
\text { choic }\end{array}$ & $\begin{array}{l}\text { Not re- } \\
\text { corded }\end{array}$ & $\begin{array}{l}\text { Adenocarcinoma, } \\
\text { recurrent }\end{array}$ & $\begin{array}{l}\text { No compli- } \\
\text { cation }\end{array}$ & $\begin{array}{l}\text { Neoadjuvant chemo- } \\
\text { therapy, radiation, } \\
\text { surgical resection } \\
\text { Deceased 5/20/08 }\end{array}$ & No \\
\hline 2 & $58 / F$ & $\begin{array}{l}\text { CT: presacral mass } \\
\text { PET: presacral mass } \\
\text { enhancement }\end{array}$ & $\begin{array}{l}\text { Hypoe- } \\
\text { choic }\end{array}$ & $\begin{array}{l}\text { Not re- } \\
\text { corded }\end{array}$ & $\begin{array}{l}\text { Adenocarcinoma, } \\
\text { recurrent }\end{array}$ & $\begin{array}{l}\text { No compli- } \\
\text { cation }\end{array}$ & $\begin{array}{l}\text { Resection of recur- } \\
\text { rence } 4 / 10 / 06 \text {. } \\
\text { Post op. CVA } \\
\text { dehiscence w eviscera- } \\
\text { tion } \\
\text { Deceased } 10 / 4 / 06\end{array}$ & No \\
\hline 3 & $44 / M$ & $\begin{array}{l}\text { CT: presacral mass } \\
\text { PET: negative }\end{array}$ & $\begin{array}{l}\text { Heteroge- } \\
\text { neous }\end{array}$ & $\begin{array}{l}\text { Not re- } \\
\text { corded }\end{array}$ & $\begin{array}{l}\text { Atypical glandular } \\
\text { cells with abun- } \\
\text { dance of mucous }\end{array}$ & $\begin{array}{l}\text { No compli- } \\
\text { cation }\end{array}$ & $\begin{array}{l}\text { Spontaneous reces- } \\
\text { sion of presacral mass. } \\
\text { Pulmonary metastasis } \\
\text { s/p chemotherapy/re- } \\
\text { section }\end{array}$ & Yes \\
\hline 4 & $80 / \mathrm{F}$ & CT: presacral mass & $\begin{array}{l}\text { Heteroge- } \\
\text { neous }\end{array}$ & $\begin{array}{l}\text { Not re- } \\
\text { corded }\end{array}$ & Myolipoma & $\begin{array}{l}\text { No compli- } \\
\text { cation }\end{array}$ & Stable repeat imaging & Yes \\
\hline 5 & $36 / \mathrm{F}$ & $\mathrm{CT}$ : presacral mass & $\begin{array}{l}\text { Heteroge- } \\
\text { neous }\end{array}$ & $\begin{array}{l}22- \\
\text { gauge } \\
\text { needle }\end{array}$ & $\begin{array}{l}\text { Anucleated squa- } \\
\text { mous cells and } \\
\text { rare spindled cells } \\
\text { favoring teratoma }\end{array}$ & $\begin{array}{l}\text { Perirectal } \\
\text { abscess }\end{array}$ & $\begin{array}{l}\text { Successful I\&D of peri- } \\
\text { rectal abscess } 2 / 2 \text { to } \\
\text { infected biopsy of sa- } \\
\text { cral teratoma } \\
\text { Lost to follow up }\end{array}$ & $\begin{array}{l}\text { Not ap- } \\
\text { plicable }\end{array}$ \\
\hline 6 & $61 / M$ & $\begin{array}{l}\text { CT presacral mass } \\
\text { PET: rising SUV of } \\
\text { presacral mass. }\end{array}$ & $\begin{array}{l}\text { Heteroge- } \\
\text { neous }\end{array}$ & $\begin{array}{l}22- \\
\text { gauge } \\
\text { needle }\end{array}$ & $\begin{array}{l}\text { Adenocarcinoma, } \\
\text { recurrent }\end{array}$ & $\begin{array}{l}\text { No compli- } \\
\text { cation }\end{array}$ & Unknown & $\begin{array}{l}\text { Not ap- } \\
\text { plicable }\end{array}$ \\
\hline 7 & $48 / \mathrm{M}$ & $\begin{array}{l}\text { MRI/CT: presacral } \\
\text { mass } \\
\text { PET: rising SUV of } \\
\text { presacral mass }\end{array}$ & $\begin{array}{l}\text { Hypoe- } \\
\text { choic }\end{array}$ & $\begin{array}{l}22- \\
\text { gauge } \\
\text { needle }\end{array}$ & $\begin{array}{l}\text { Adenocarcinoma, } \\
\text { recurrent }\end{array}$ & $\begin{array}{l}\text { No compli- } \\
\text { cation }\end{array}$ & Unknown & $\begin{array}{l}\text { Not ap- } \\
\text { plicable }\end{array}$ \\
\hline 8 & $57 / F$ & $\begin{array}{l}\text { CT: } 1.5-\mathrm{cm} \text { node in } \\
\text { sigmoid mesocolon } \\
\text { PET: no evidence of } \\
\text { tumor from previous } \\
\text { colorectal cancer }\end{array}$ & $\begin{array}{l}\text { Hypoe- } \\
\text { choic node }\end{array}$ & $\begin{array}{l}25- \\
\text { gauge } \\
\text { needle }\end{array}$ & $\begin{array}{l}\text { Benign lymphoid } \\
\text { hyperplasia }\end{array}$ & $\begin{array}{l}\text { No compli- } \\
\text { cation }\end{array}$ & $\begin{array}{l}\text { Reoccurrence of colo- } \\
\text { rectal cancer with me- } \\
\text { tastatic disease }\end{array}$ & $\begin{array}{l}\text { Yes (Sur- } \\
\text { gery a- } \\
\text { voided at } \\
\text { time of } \\
\text { RUS- } \\
\text { FNA) }\end{array}$ \\
\hline 9 & $43 / F$ & $\begin{array}{l}\text { MRI: multilocular } \\
\text { presacral cystic mass } \\
\text { without worrisome } \\
\text { enhancement. }\end{array}$ & $\begin{array}{l}\text { Heteroge- } \\
\text { neous }\end{array}$ & $\begin{array}{l}25- \\
\text { gauge } \\
\text { needle }\end{array}$ & $\begin{array}{l}\text { Mucous with be- } \\
\text { nign appearing } \\
\text { epithelial cells, } \\
\text { overall non-diag- } \\
\text { nostic }\end{array}$ & $\begin{array}{l}\text { No compli- } \\
\text { cation }\end{array}$ & $\begin{array}{l}\text { Presacral cysic mass: } \\
\text { Coccygectomy, partial } \\
\text { sacrectomy, presacral } \\
\text { mass resection. } \\
\text { Path returned retro- } \\
\text { rectal cystic hamarto- } \\
\text { ma. }\end{array}$ & No \\
\hline 10 & $48 / \mathrm{F}$ & $\begin{array}{l}\mathrm{CT} \text { : rectal mass } \\
\text { PET: large hypermeta- } \\
\text { bolic mass at the rec- } \\
\text { tosigmoid junction } \\
\text { with hypermetabolic } \\
\text { retroperitoneal left } \\
\text { iliac chain lymph } \\
\text { nodes concerning for } \\
\text { metastatic nodal } \\
\text { spread. }\end{array}$ & $\begin{array}{l}\text { Hypoe- } \\
\text { choic }\end{array}$ & $\begin{array}{l}22-\text { and } \\
25- \\
\text { gauge } \\
\text { needle }\end{array}$ & $\begin{array}{l}\text { Squamous cell } \\
\text { carcinoma }\end{array}$ & $\begin{array}{l}\text { No compli- } \\
\text { cation }\end{array}$ & $\begin{array}{l}\text { T3N2 stage IIIB anal/ } \\
\text { rectal squamous cell } \\
\text { carcinoma s/p chemo/ } \\
\text { radiation with com- } \\
\text { plete response }\end{array}$ & Yes \\
\hline
\end{tabular}


- Table 1 (Continuation)

\begin{tabular}{|c|c|c|c|c|c|c|c|c|}
\hline & $\begin{array}{l}\text { Age/ } \\
\text { Sex }\end{array}$ & $\begin{array}{l}\text { Radiographic } \\
\text { findings }\end{array}$ & $\begin{array}{l}\text { Overall } \\
\text { U/S ap- } \\
\text { pearance }\end{array}$ & $\begin{array}{l}\text { Fine- } \\
\text { needle } \\
\text { gauge }\end{array}$ & $\begin{array}{l}\text { Pathology ob- } \\
\text { tained from FNA }\end{array}$ & $\begin{array}{l}\text { Compli- } \\
\text { cations }\end{array}$ & Outcome & $\begin{array}{l}\text { Surgery } \\
\text { avoided } \\
\text { (Yes/No) }\end{array}$ \\
\hline 11 & $62 / \mathrm{F}$ & CT: presacral mass & $\begin{array}{l}\text { Heteroge- } \\
\text { neous }\end{array}$ & $\begin{array}{l}22- \\
\text { gauge } \\
\text { needle }\end{array}$ & $\begin{array}{l}\text { Numerous anucle- } \\
\text { ate and nucleated } \\
\text { squamous, colum- } \\
\text { nar cells, and cho- } \\
\text { lesterol crystals } \\
\text { DDx: teratoma, } \\
\text { epidermal cyst } \\
\text { and tailgut cyst }\end{array}$ & $\begin{array}{l}\text { Rectal pain; } \\
\text { sepsis; pre- } \\
\text { sacral ab- } \\
\text { scess with } \\
\text { drainage, } \\
\text { hemorrha- } \\
\text { gic stroke, } \\
\text { ARF }\end{array}$ & I\&D & No \\
\hline 12 & $64 / M$ & $\begin{array}{l}\text { MRI: suggestive of } \\
\text { duplication cyst }\end{array}$ & $\begin{array}{l}\text { Heteroge- } \\
\text { neous }\end{array}$ & $\begin{array}{l}22- \\
\text { gauge } \\
\text { needle }\end{array}$ & $\begin{array}{l}\text { Benign squamous } \\
\text { epithelial cells and } \\
\text { crystals. }\end{array}$ & $\begin{array}{l}\text { No compli- } \\
\text { cation }\end{array}$ & Unknown & Yes \\
\hline 13 & $34 / \mathrm{F}$ & MRI: rectal mass & $\begin{array}{l}\text { Hypoe- } \\
\text { choic }\end{array}$ & $\begin{array}{l}\text { Not re- } \\
\text { corded }\end{array}$ & $\begin{array}{l}\text { GIST, epithelioid } \\
\text { type with atypia }\end{array}$ & $\begin{array}{l}\text { No compli- } \\
\text { cation }\end{array}$ & $\begin{array}{l}\text { Hysterectomy and } \\
\text { partial vaginectomy } \\
\text { for what was originally } \\
\text { thought to be a GIST; } \\
\text { ultimately turned out } \\
\text { to be endometrial de- } \\
\text { posit in cul-de-sac. }\end{array}$ & No \\
\hline 14 & $53 / F$ & $\begin{array}{l}\mathrm{CT} \text { : thickened rectal } \\
\text { wall }\end{array}$ & $\begin{array}{l}\text { Hypoe- } \\
\text { choic }\end{array}$ & $\begin{array}{l}22- \\
\text { gauge } \\
\text { needle }\end{array}$ & $\begin{array}{l}\text { Adenocarcinoma, } \\
\text { recurrent }\end{array}$ & $\begin{array}{l}\text { No compli- } \\
\text { cation }\end{array}$ & $\begin{array}{l}\text { Received neoadjuvant } \\
\text { chemotherapy/radia- } \\
\text { tion, Surgical resection }\end{array}$ & No \\
\hline 15 & $31 / F$ & $\begin{array}{l}\text { No prior imaging re- } \\
\text { ported }\end{array}$ & $\begin{array}{l}\text { Hypoe- } \\
\text { choic }\end{array}$ & $\begin{array}{l}22- \\
\text { gauge } \\
\text { needle }\end{array}$ & $\begin{array}{l}\text { Colorectal-type } \\
\text { epithelium and } \\
\text { abundant mucus }\end{array}$ & $\begin{array}{l}\text { No compli- } \\
\text { cation }\end{array}$ & Lost to follow up & Yes \\
\hline 16 & $59 / \mathrm{M}$ & $\mathrm{CT}$ : rectosigmoid mass & $\begin{array}{l}\text { Hypoe- } \\
\text { choic node }\end{array}$ & $\begin{array}{l}\text { Not re- } \\
\text { corded }\end{array}$ & $\begin{array}{l}\text { Adenocarcinoma, } \\
\text { recurrent }\end{array}$ & $\begin{array}{l}\text { No compli- } \\
\text { cation }\end{array}$ & $\begin{array}{l}\text { Neoadjuvant chemo- } \\
\text { therapy, resection }\end{array}$ & No \\
\hline 17 & $57 / \mathrm{M}$ & $\begin{array}{l}\text { PET/CT: presacral soft } \\
\text { tissue lesion concern- } \\
\text { ing for local recurrence } \\
\text { vs inflammation }\end{array}$ & $\begin{array}{l}\text { Hypoe- } \\
\text { choic }\end{array}$ & $\begin{array}{l}25- \\
\text { gauge } \\
\text { needle }\end{array}$ & $\begin{array}{l}\text { Inflammation con- } \\
\text { sistent with ab- } \\
\text { scess }\end{array}$ & $\begin{array}{l}\text { No compli- } \\
\text { cation }\end{array}$ & $\begin{array}{l}\text { Treated with antibio- } \\
\text { tics }\end{array}$ & Yes \\
\hline 18 & $30 / \mathrm{M}$ & $\begin{array}{l}\mathrm{CT} \text { : circumscribed soft } \\
\text { tissue/fluid density } \\
\text { structure in the presa- } \\
\text { cral space }\end{array}$ & $\begin{array}{l}\text { Hypoe- } \\
\text { choic }\end{array}$ & $\begin{array}{l}22- \\
\text { gauge } \\
\text { needle }\end{array}$ & $\begin{array}{l}\text { Benign squamous } \\
\text { epithelial cells } \\
\text { query cystic terato- } \\
\text { ma }\end{array}$ & $\begin{array}{l}\text { Rectal pain } \\
\text { and infected } \\
\text { presacral } \\
\text { mass-presa- } \\
\text { cral abscess }\end{array}$ & $\begin{array}{l}\text { Excision of infected } \\
\text { presacral mass: rup- } \\
\text { tured dermoid cyst } \\
\text { with prominent mela- } \\
\text { nin pigmentation }\end{array}$ & No \\
\hline 19 & $63 / M$ & $\begin{array}{l}\mathrm{CT} \text { : thickening of the } \\
\text { mid and distal esopha- } \\
\text { gus consistent with } \\
\text { history of esophageal } \\
\text { carcinoma. Soft tissue } \\
\text { enhancement anterior } \\
\text { to the rectum. } \\
\text { PET: soft tissue lesion } \\
\text { in the pelvis, between } \\
\text { the urinary bladder } \\
\text { and rectum shows hy- } \\
\text { permetabolic activity } \\
\text { with a maximum SUV } \\
\text { of } 4 \text {. Concerning for a } \\
\text { peritoneal metastatic } \\
\text { deposit. }\end{array}$ & $\begin{array}{l}\text { Hypoe- } \\
\text { choic }\end{array}$ & $\begin{array}{l}22- \\
\text { gauge } \\
\text { needle }\end{array}$ & $\begin{array}{l}\text { Amorphous mate- } \\
\text { rial of uncertain } \\
\text { type and a few } \\
\text { clusters of pig- } \\
\text { ment-containing } \\
\text { epithelial cells. No } \\
\text { malignancy is } \\
\text { identified in this } \\
\text { material. The find- } \\
\text { ings raise the pos- } \\
\text { sibility of seminal } \\
\text { vesicle sampling. }\end{array}$ & $\begin{array}{l}\text { No compli- } \\
\text { cation }\end{array}$ & $\begin{array}{l}\text { Progressive esopha- } \\
\text { geal cancer }\end{array}$ & Yes \\
\hline 20 & $37 / \mathrm{M}$ & $\begin{array}{l}\mathrm{CT} / \mathrm{PET} \text { : perirectal } \\
\text { mass }\end{array}$ & $\begin{array}{l}\text { Hypoe- } \\
\text { choic }\end{array}$ & $\begin{array}{l}22-\text { and } \\
25- \\
\text { gauge } \\
\text { needle }\end{array}$ & $\begin{array}{l}\text { Anucleated squa- } \\
\text { mous cells and rare } \\
\text { benign glandular } \\
\text { cells. No malig- } \\
\text { nancy identified. }\end{array}$ & $\begin{array}{l}\text { Perirectal } \\
\text { abscess }\end{array}$ & $\begin{array}{l}\text { Transrectal drainage } \\
\text { of perirectal abscess }\end{array}$ & Yes \\
\hline
\end{tabular}


- Table 1 (Continuation)

\begin{tabular}{|c|c|c|c|c|c|c|c|c|}
\hline & $\begin{array}{l}\text { Agel } \\
\text { Sex }\end{array}$ & $\begin{array}{l}\text { Radiographic } \\
\text { findings }\end{array}$ & $\begin{array}{l}\text { Overall } \\
\text { U/S ap- } \\
\text { pearance }\end{array}$ & $\begin{array}{l}\text { Fine- } \\
\text { needle } \\
\text { gauge }\end{array}$ & $\begin{array}{l}\text { Pathology ob- } \\
\text { tained from FNA }\end{array}$ & $\begin{array}{l}\text { Compli- } \\
\text { cations }\end{array}$ & Outcome & $\begin{array}{l}\text { Surgery } \\
\text { avoided } \\
\text { (Yes/No) }\end{array}$ \\
\hline 21 & $75 / F$ & $\begin{array}{l}\text { PET: rectal hypermeta- } \\
\text { bolic area }\end{array}$ & $\begin{array}{l}\text { Hypoe- } \\
\text { choic }\end{array}$ & $\begin{array}{l}22- \\
\text { gauge } \\
\text { needle }\end{array}$ & $\begin{array}{l}\text { Marked acute in- } \\
\text { flammation consis- } \\
\text { tent with benign } \\
\text { reactive process. } \\
\text { Negative for malig- } \\
\text { nancy. }\end{array}$ & $\begin{array}{l}\text { No compli- } \\
\text { cation }\end{array}$ & $\begin{array}{l}\text { No recurrence to date } \\
\text { of previous diagnosed } \\
\text { colorectal cancer }\end{array}$ & Yes \\
\hline 22 & $53 / F$ & $\begin{array}{l}\mathrm{CT} \text { : irregular enhanc- } \\
\text { ing mass along the } \\
\text { posterior right vaginal } \\
\text { wall adjacent to the } \\
\text { rectum. }\end{array}$ & $\begin{array}{l}\text { Hypoe- } \\
\text { choic }\end{array}$ & $\begin{array}{l}25- \\
\text { gauge } \\
\text { needle }\end{array}$ & $\begin{array}{l}\text { Poorly differenti- } \\
\text { ated squamous } \\
\text { cell carcinoma. }\end{array}$ & $\begin{array}{l}\text { No compli- } \\
\text { cation }\end{array}$ & $\begin{array}{l}\text { T2N0 anal canal can- } \\
\text { cer. Definitive che- } \\
\text { moradiation }\end{array}$ & Yes \\
\hline 23 & $19 / F$ & $\begin{array}{l}\mathrm{CT} / \mathrm{RUS} \text { : lymph node } \\
\text { seen }\end{array}$ & $\begin{array}{l}\text { Hypoe- } \\
\text { choic }\end{array}$ & $\begin{array}{l}25- \\
\text { gauge } \\
\text { needle }\end{array}$ & $\begin{array}{l}\text { Benign lymphoid } \\
\text { hyperplasia }\end{array}$ & $\begin{array}{l}\text { No compli- } \\
\text { cation }\end{array}$ & $\begin{array}{l}\text { Lynch positive family; } \\
\text { neoadjuvant chemo- } \\
\text { therapy, radiation ther- } \\
\text { apy, and protocolect- } \\
\text { omy }\end{array}$ & No \\
\hline 24 & $54 / M$ & $\begin{array}{l}\mathrm{CT} \text { : large calcified } \\
\text { mass in the pelvis with } \\
\text { erosion of portions of } \\
\text { the ischium and the } \\
\text { superior pubic ramus. }\end{array}$ & $\begin{array}{l}\text { Heteroge- } \\
\text { neous }\end{array}$ & $\begin{array}{l}25- \\
\text { gauge } \\
\text { needle }\end{array}$ & $\begin{array}{l}\text { Spindle cell neo- } \\
\text { plasm. Immuno- } \\
\text { histochemical } \\
\text { profile in keeping } \\
\text { with a diagnosis of } \\
\text { a primitive neu- } \\
\text { roectodermal tu- } \\
\text { mor/soft tissue } \\
\text { sarcoma }\end{array}$ & $\begin{array}{l}\text { No compli- } \\
\text { cation }\end{array}$ & $\begin{array}{l}\text { Pulmonary metastasis; } \\
\text { received chemother- } \\
\text { apy }\end{array}$ & Yes \\
\hline 25 & $46 / F$ & $\begin{array}{l}\mathrm{CT} \text { : thickened sigmoid } \\
\text { and adnexal mass }\end{array}$ & $\begin{array}{l}\text { Hypoe- } \\
\text { choic }\end{array}$ & $\begin{array}{l}25- \\
\text { gauge } \\
\text { needle }\end{array}$ & $\begin{array}{l}\text { Atypical glandular } \\
\text { cells. No malig- } \\
\text { nancy is identified }\end{array}$ & $\begin{array}{l}\text { No compli- } \\
\text { cation }\end{array}$ & $\begin{array}{l}\text { Mass over } 2 \mathrm{~cm} \text { under- } \\
\text { went sigmoid resec- } \\
\text { tion and pathology re- } \\
\text { vealed endometriosis }\end{array}$ & No \\
\hline 26 & $46 / M$ & $\begin{array}{l}\mathrm{CT} \text { : bowel thickening } \\
\text { at ileoanal anastomo- } \\
\text { sis }\end{array}$ & $\begin{array}{l}\text { Heteroge- } \\
\text { neous }\end{array}$ & $\begin{array}{l}25- \\
\text { gauge } \\
\text { needle }\end{array}$ & $\begin{array}{l}\text { Anus biopsy: tub- } \\
\text { ular adenoma. } \\
\text { FNA: abundant } \\
\text { amorphous debris, } \\
\text { pigmented gland- } \\
\text { ular cells and sper- } \\
\text { matozoa consis- } \\
\text { tent w seminal ve- } \\
\text { sicle sampling. No } \\
\text { neoplasia }\end{array}$ & $\begin{array}{l}\text { No compli- } \\
\text { cation }\end{array}$ & Continued follow up & Yes \\
\hline 27 & $73 / \mathrm{M}$ & $\begin{array}{l}\text { CT: pelvic mass PET: } \\
\text { large hypermetabolic } \\
\text { mass along the right } \\
\text { pelvic sidewall along } \\
\text { with a smaller hyper- } \\
\text { metabolic nodule } \\
\text { slightly more superior } \\
\text { are consistent with re- } \\
\text { currence of disease in } \\
\text { this patient with a his- } \\
\text { tory of bladder cancer. }\end{array}$ & $\begin{array}{l}\text { Hypoe- } \\
\text { choic }\end{array}$ & $\begin{array}{l}\text { Not re- } \\
\text { corded }\end{array}$ & $\begin{array}{l}\text { Metastatic bladder } \\
\text { cancer }\end{array}$ & $\begin{array}{l}\text { No compli- } \\
\text { cation }\end{array}$ & $\begin{array}{l}\text { Continued follow-up } \\
\text { Received chemother- } \\
\text { apy }\end{array}$ & Yes \\
\hline
\end{tabular}

U/S, ultrasound; FNA, fine-needle aspiration; CT, computed tomography; PET, positron emission tomography; I\&D, incision and drainage; SUV, standard uptake value; MRI, magnetic resonance imaging; ARF, acute renal failure; GIST, gastrointestinal stromal tumor; RUS, rectal ultrasound 
> Table 2 RUS-FNA findings.

\begin{tabular}{|c|c|c|c|c|}
\hline Lesion & Size $(\mathrm{cm})^{1}$ & Avg number of passes & Findings & Complication \\
\hline $\begin{array}{l}\text { Presacral mass } \\
(n=12)\end{array}$ & $\begin{array}{l}4.2 \\
\text { (range } 2.5-7.6 \text { ) }\end{array}$ & $\begin{array}{l}2.6 \\
\text { (range } 1-5 \text { ) }\end{array}$ & $\begin{array}{l}\text { Adenocarcinoma }(n=4) \\
\text { Cystic lesion }(n=3) \\
\text { Other benign cells }(n=2) \\
\text { Myelolipoma }(n=1) \\
\text { Sarcoma }(n=1) \\
\text { Non-diagnostic }(n=1)\end{array}$ & $\begin{array}{l}3 \\
25 \%\end{array}$ \\
\hline $\begin{array}{l}\text { Perirectal abnormality } \\
(\mathrm{n}=12)\end{array}$ & $\begin{array}{l}2.7 \\
\text { (range 1.3-4.5) }\end{array}$ & $\begin{array}{l}2.9 \\
\text { (range } 1-5 \text { ) }\end{array}$ & $\begin{array}{l}\text { Adenocarcinoma }(n=2) \\
\text { Squamous cell carcinoma }(n=2) \\
\text { Other benign cells }(n=4) \\
\text { Cystic lesion }(n=1) \\
\text { Seminal vesicle }(n=1) \\
\text { Non-diagnostic }(n=2)\end{array}$ & $\begin{array}{l}1 \\
8 \%\end{array}$ \\
\hline $\begin{array}{l}\text { Perirectal node } \\
(n=2)\end{array}$ & $\begin{array}{l}0.95 \\
\text { (range } 0.9-1.0 \text { ) }\end{array}$ & $\begin{array}{l}4 \\
\text { (both 4) }\end{array}$ & Benign lymphoid hyperplasia $(n=2)$ & 0 \\
\hline $\begin{array}{l}\text { Pelvic mass } \\
(n=1)\end{array}$ & $\begin{array}{l}4.7 \\
\text { (range 4.7) }\end{array}$ & $\begin{array}{l}2 \\
\text { (range 2) }\end{array}$ & $\begin{array}{l}\text { Urothelial Bladder Cancer } \\
(n=1)\end{array}$ & 0 \\
\hline
\end{tabular}

with EUS-FNA from the upper gastrointestinal tract. Studies show that upper gastrointestinal FNAs appear to have fewer complication rates compared to lower gastrointestinal FNAs. The reported complication rate performing an endoscopic ultrasound (EUS)-FNA of the pancreas is $1 \%$ to $2.5 \%[18,19]$. In comparison, a study evaluating adverse events (AEs) in lower gastrointestinal EUS-FNA reported AEs in $20.6 \%$ of cases, mostly in the form of bleeding and pain, with $5.6 \%$ of those events being serious [20]. Interestingly, few infectious complications have been reported in upper and lower gastrointestinal FNAs $[19,20]$. RUS-FNA has been proven a safe method for tissue sampling, with incidence of bacteremia similar to or less than that seen in diagnostic colonoscopy [21]. A lesion's characteristics appear to contribute to risk of complications. An increased risk of febrile episodes or sepsis has been observed in upper gastrointestinal FNAs of cystic lesions $[20,22,23]$. It is unclear why our study showed such a high rate of infectious complications. Two out of the four lesions were heterogeneous and not purely solid, which may have increased the likelihood of infectious complications. Studies suggest that biopsy of presacral lesions does not add to the surgical strategy and that biopsies vary in accuracy [24]. However, the utility of tissue sampling has been increased with improved techniques and preoperative treatments. Presacral lesions such as Ewing sarcomas, osteosarcomas, lymphomas, and fibrous tumors are examples of lesions that could benefit from neoadjuvant therapy [25]. Patients' medical treatment would be improved by preoperative biopsy of such lesions. Certainly, continued use of prophylactic antibiotics, minimization of needle passages, and use of experienced endosonographers can minimize complications in RUSFNA.
To our knowledge, there is little data in the literature outlining the diagnostic yield or complication rate of CT-guided biopsy via a transgluteal approach for perirectal, presacral, or pelvic lesions. Success rates with CT-guided prostate biopsies have been upward of $95 \%$ to $97 \%$ and a study performed in 2003 showed a $93 \%$ diagnostic yield of pelvic lesions by an extraperitoneal approach [26-28] Despite this lack of data, CT-guided percutaneous biopsy has been described as being an appropriate method for biopsy of lesions located in perirectal, presacral, and posterior pelvic regions and superior in distant or metastatic disease $[29,30]$. However, there are disadvantages to transgluteal CT-guided FNA, including pain, patient discomfort due to lying in prone position for an extended period of time, and risk of gluteal vessel, sciatic nerve, and sacral plexus injury [29]. It can be argued that RUS-FNA may palliate many of the aforementioned disadvantages by minimizing pain, allowing patients to lie in the left lateral decubitus position, and providing the proceduralist with closer anatomic proximity to lesions to accurately obtain a tissue diagnosis and improve staging of primary/recurrent malignancies.

Our study is limited by a small sample size of 27 patients and the retrospective design. Admittedly, there are concerns about later complications possibly being missed as patients could have gone to their local community hospital or physician rather than returning to our facility. All biopsies could not be corroborated with surgical specimen pathology because results of FNA biopsies dictated medical decision-making and prevented some patients from having a surgical intervention. Also, our study was not a comparative study to differentiate the diagnostic yield between CT-guided biopsy versus RUS-FNA. Ideally, these two imaging modalities should have much larger studies for comparison in diagnostic yield and complication rates. 


\section{Conclusion}

In summary, RUS-FNA is an accurate and relatively safe method for obtaining tissue diagnosis of presacral, perirectal, and pelvic lesions when performed by experienced endosonographers. While the diagnostic yield of RUS-FNA is high and the potential to affect clinical decision-making is real, the risk of complication is not negligible. RUS-FNA should only be performed if the result will substantially alter clinical management, and the decision to perform RUS-FNA should be made by a multidisciplinary team.

\section{Competing interests}

Girish Mishra - Consultant, Cook Medical, Pentax Medical. Norman Clark - none. Landon Brown - none. Jason Conway - Consultant, Cook Medical, Pentax Medical

\section{References}

[1] Siegel RL, Miller KD, Jemal A. Cancer statistics, 2016. CA Cancer J Clin 2016; 66: 7-30

[2] van Gijn W, Marijnen CA, Nagtegaal ID et al. Preoperative radiotherapy combined with total mesorectal excision for resectable rectal cancer: 12-year follow-up of the multicentre, randomised controlled TME trial. Lancet Oncol 2011; 12: 575- 582

[3] Taylor FG, Quirke P, Heald R] et al. Preoperative high-resolution magnetic resonance imaging can identify good prognosis stage I, II, and III rectal cancer best managed by surgery alone: a prospective, multicenter, European study. Ann Surg 2011; 253: 711 - 719

[4] Bernstein TE, Endreseth BH, Romundstad P et al. Circumferential resection margin as a prognostic factor in rectal cancer. Br J Surg 2009; 96: $1348-1357$

[5] Marin G, Suárez J, Vera R et al. Local recurrence after five years is associated with preoperative chemoradiotherapy treatment in patients diagnosed with stage II and III rectal cancer. Int J Surg 2017; 44: $15-20$

[6] Harewood GC. Assessment of clinical impact of endoscopic ultrasound on rectal cancer. Am J Gastroenterol 2004; 99: 623-627

[7] Kwok H, Bissett IP, Hill GL. Preoperative staging of rectal cancer. Int ] Colorectal Dis 2000; 15: 9-20

[8] Guinet C, Buy JN, Ghossain MA et al. Comparison of magnetic resonance imaging and computed tomography in the preoperative staging of rectal cancer. Arch Surg 1990; 125: 385 - 388

[9] Rifkin MD, Ehrlich SM, Marks G. Staging of rectal carcinoma: prospective comparison of endorectal US and CT. Radiology 1989; 170: $319-322$

[10] Thaler W, Watzka S, Martin F et al. Preoperative staging of rectal cancer by endoluminal ultrasound vs. magnetic resonance imaging. Preliminary results of a prospective, comparative study. Dis Colon Rectum 1994; 37: 1189-1193

[11] Soh JS, Lee HS, Lee $S$ et al. The clinical usefulness of endoscopic ultrasound-guided fine needle aspiration and biopsy for rectal and perirectal lesions. Intest Res 2015; 13: 135-144
[12] Fernández-Esparrach G, Alberghina N, Subtil JC et al. Endoscopic ultrasound-guided fine needle aspiration is highly accurate for the diagnosis of perirectal recurrence of colorectal cancer. Dis Colon Rectum 2015; 58: $469-473$

[13] Rzouq F, Brown J, Fan F et al. The utility of lower endoscopic ultrasound-guided fine needle aspiration for the diagnosis of benign and malignant pelvic diseases. J Clin Gastroenterol 2014; 48: 127 -130

[14] Mohamadnejad M, Al-Haddad MA, Sherman S et al. Utility of EUSguided biopsy of extramural pelvic masses. Gastrointest Endosc 2012; 75: $146-151$

[15] Maleki Z, Erozan Y, Geddes S et al. Endorectal ultrasound-guided fineneedle aspiration: a useful diagnostic tool for perirectal and intraluminal lesions. Acta Cytol 2013; 57: 9-18

[16] Gleeson FC, Larson DW, Dozois EJ et al. Local recurrence detection following transanal excision facilitated by EUS-FNA. Hepatogastroenterology 2012; 59: $1102-1107$

[17] Hassan GM, Paquin SC, Albadine R et al. Endoscopic ultrasound-guided FNA of pelvic lesions: A large single-center experience. Cancer $\mathrm{Cy}$ topathol 2016; 124: 836-841

[18] Eloubeidi MA, Tamhane A, Varadarajulu S et al. Frequency of major complications after EUS-guided FNA of solid pancreatic masses: a prospective evaluation. Gastrointest Endosc 2006; 63: 622-629

[19] Adler DG, Jacobson BC, Davila RE et al. ASGE guideline: complications of EUS. Gastrointest Endosc 2005; 61: 8-12

[20] Levy M], Abu Dayyeh BK, Fujii LL et al. Prospective evaluation of adverse events following lower gastrointestinal tract EUS FNA. Am J Gastroenterol 2014; 109: 676-685

[21] Levy MJ, Norton ID, Clain JE et al. Prospective study of bacteremia and complications with EUS FNA of rectal and perirectal lesions. Clin Gastroenterol Hepatol 2007; 5: 684-689

[22] Wiersema M], Vilmann P, Giovannini M et al. Endosonography-guided fine-needle aspiration biopsy: diagnostic accuracy and complication assessment. Gastroenterology 1997; 112: 1087-1095

[23] Lee LS, Saltzman JR, Bounds BC et al. EUS-guided fine needle aspiration of pancreatic cysts: a retrospective analysis of complications and their predictors. Clin Gastroenterol Hepatol 2005; 3: 231 - 236

[24] Toh JW, Morgan M. Management approach and surgical strategies for retrorectal remours: a systematic review. Colorectal Dis 2016; 18: $337-350$

[25] Patel N, Maturen KE, Kaza RK et al. Imaging of presacral masses a multidisciplinary approach. Br J Radiol 2016; 89: 20150698

[26] Olson MC, Atwell TD, Mynderse LA et al. CT-guided transgluteal biopsy for systematic sampling of the prostate in patients without rectal access: a 13-year single-center experience. Eur Radiol 2017; 27: $3326-3332$

[27] Goenka AH, Remer EM, Veniero JC et al. CT-guided transgluteal biopsy for systematic random sampling of the prostate in patients without rectal access. AJR AM J Roentgenol 2015; 205: 578-583

[28] Gupta S, Madoff DC, Ahrar K et al. CT-guided needle biopsy of deep pelvic lesions by extraperitoneal approach through iliopsoas muscle. Cardiovasc Intervent Radiol 2003; 26: 534-538

[29] Gupta S, Nguyen HL, Morello FA Jr et al. Various approaches for CTguided percutaneous biopsy of deep pelvic lesions: anatomic and technical considerations. Radiographics 2004; 24: 175-189

[30] Siddiqui AA, Fayiga Y, Huerta S. The role of endoscopic ultrasound in the evaluation of rectal cancer. Int Semin Surg Oncol 2006; 18: 36 